\title{
CORRELAÇÃO ENTRE AS CAPACIDADES FÍSICAS BÁSICAS E O ÍNDICE DE CAPACIDADE DE TRABALHO EM BOMBEIROS DO ESTADO DO RIO DE JANEIRO
}

\section{Correlation between basic physical capacities and the work capacity index in firefighters of the state of rio de janeiro}

\author{
Cristiano Marcelino', Roberto Simão', Raphael Guimarães ${ }^{1}$, Belmiro Freitas de Salles ${ }^{1}$, Juliano Spineti ${ }^{1}$ \\ ${ }^{1}$ Universidade Federal do Rio de Janeiro. Escola de Educação Física e Desportos. Rio de Janeiro - RJ - Brasil.
}

\begin{abstract}
Resumo: O propósito desse estudo foi investigar as características físicas básicas do Bombeiro Militar do Corpo de Bombeiros Militar do Estado do Rio de Janeiro (CBMERJ) e correlacionar o nível de aptidão física com o índice de capacidade de trabalho (ICT). A amostra foi composta por 14 voluntários masculinos (33 \pm 4 anos, 1,79 \pm 0,07 metros, 83,4 $\pm 11,6 \mathrm{~kg}$ ), alunos do Curso de Monitor de Educação Física do CBMERJ. Os dados foram coletados em três dias não consecutivos ao longo de uma semana. No primeiro dia, foram realizadas a anamnese geral e a aplicação do questionário PAR-Q e do questionário para avaliar o ICT, e as medidas antropométricas. O segundo dia foi utilizado para a realização dos testes de aptidão musculoesquelética, tais como: flexibilidade, impulsão horizontal, resistência muscular para membros superiores e abdômen e força isométrica. $\mathrm{O}$ terceiro dia de teste foi destinado para o teste de resistência aeróbia (corrida de 2.400 metros). Para análise estatística descritiva, o tratamento empregado foi de medidas de tendência central e medidas de dispersão, valores mínimos e máximos alcançados, além de uma TABELA de percentis por qualidade física. Para verificar a correlação entre o somatório do score-t e o ICT foi utilizada a equação de Produto-Momento de Pearson, para tal foi utilizado o programa Estatística versão 7.0. Não foi encontrada correlação entre o ICT e o nível de aptidão física, os participantes apresentaram bons níveis de aptidão física nos testes de resistência aeróbia, impulsão horizontal, flexão de braços e abdominal, entretanto não ocorreu o mesmo para a verificação da flexibilidade e da força isométrica em barra fixa. Em conclusão, O ICT não mostrou ser um método adequado para quantificar a capacidade de trabalho desses profissionais.

Palavras-chaves: Exercício, Testes de Exercícios, Força Muscular, Índice de Capacidade de Trabalho, Atividade Física, Bombeiros.
\end{abstract}

\begin{abstract}
The purpose of this experiment was to investigate the basic physical characteristics of a military firefighter of Corpo de Bombeiro Militar do Rio de Janeiro (CBMRJ) and to correlate the physical fitness level with the work capacity index (WCI). Participants of this study were 14 military firefighters ( $33 \pm 4$ years; $179 \pm 0.07 \mathrm{~cm} ; 83.4 \pm 11.6 \mathrm{~kg}$ ) students of the course for exercise coach 2006 (CMEF) of CBMERJ. Data were collected in three nonconsecutive days. On the first day, Anamnesis of the participants were executed, and the PAR-Q and WCI questionnaires were answered before the measurement of the participants' anthropometric variables. The second day was used for the accomplishment of muscle-skeletal tests. The physical capacity was measured by flexibility, muscle endurance of upper body and abdomen, and isometric strength. The third day of test was used for aerobic resistance test ( $2.400 \mathrm{~m}$ test). For descriptive statistical analysis, the treatment employed was of central trend and dispersion measures, minimum and maximum values, beyond a table of percentiles for physical quality. Pearson's equation of Product-Moment was used to verify the correlation between score-t and the $\mathrm{WCI}$, the Statistics version 7.0 program was used. In conclusion, a correlation between the $\mathrm{WCl}$ and the level of physical capacity of the firefighters of CBMERJ was not found. In accordance with the results of the basic physical capacity tests, the participants showed good levels of physical capacity in aerobic resistance, horizontal impulse, push-ups and abdominal tests. However, it did not occur the same for the verification of flexibility and the isometric test in fixed bar. In conclusion, the level of physical capacity seems not to be the basic factor for the $\mathrm{WCl}$ of these professionals.
\end{abstract}

Key words: Exercise, Exercise Tests, Muscle Strength, Work Capacity Index, Physical Activity, Firefighters.

Aceito em 12/12/2008 - Rev. Educ. Fís. 2009 Mar: 144: 36-44. Rio de Janeiro - RJ - Brasil

\section{INTRODUÇÃO}

O Corpo de Bombeiros Militar do Estado do Rio de Janeiro (CBMERJ), atualmente, com 105 unidades operacionais e um efetivo de aproximadamente
15.000 militares atua em diversas áreas da segurança pública. Dentre as mais operacionais podemos citar: combate a incêndios de todas as classes, busca e salvamento, socorro florestal e meio ambiente, remoção de cadáver e salvamento 
marítimo. Um combatente do Corpo de Bombeiros deve estar bem preparado fisicamente para atender a qualquer chamado de emergência, pois sabemos que o rápido atendimento é condição "sine qua non" no salvamento às vítimas. Devido à grande exigência física atribuída a essas atividades, o CBMERJ exige que seus integrantes tenham uma satisfatória aptidão física. Por esse motivo, são aplicadas baterias de testes físicos e análises corporais periodicamente, com o objetivo de verificar a condição morfofuncional dos militares, bem como incentivá-los à melhoria ou manutenção.

As pesquisas a respeito da caracterização do perfil físico de militares vêm crescendo nesses últimos anos, e novas conclusões, sugestões e recomendações têm sido evidenciadas, e algumas tropas já possuem algum tipo de caracterização morfofuncional ${ }^{(1,2,3,4,5,6)}$. Em geral, são analisados aqueles que possuem funções operacionais, nas quais a condição morfofuncional pode ser determinante para o sucesso da operação.

Boldori (7) avaliou 359 Bombeiros Militares do Estado de Santa Catarina, para as qualidades físicas básicas e morfológicas e as correlacionou com a capacidade de trabalho através do questionário auto-aplicável Índice de Capacidade para o Trabalho ${ }^{(8)}$. O autor concluiu através de uma análise qualitativa, que os bombeiros que possuíam sua aptidão física considerada ideal, apresentaram baixa incidência de doenças e alto Índice de Capacidade para o Trabalho. Todavia, o objetivo do estudo não foi fornecer valores normativos para a prescrição e avaliação desses militares, não utilizando instrumentos estatísticos adequados para esse tipo de avaliação, além de utilizar uma análise qualitativa para verificar tal relação, o que fornece conclusões subjetivas.

O propósito do presente estudo foi investigar as características básicas dos alunos do Curso de Monitor de Educação Física do Bombeiro Militar do CBMERJ e correlacionar o nível de aptidão física com o Índice de Capacidade de Trabalho
(ICT). Além disso, também foi objetivo do presente estudo verificar e analisar o desempenho físico dos bombeiros.

\section{METODOLOGIA}

\section{Amostra}

Participaram do estudo 14 Bombeiros Militares do sexo masculino (33 \pm 4 anos, 1,79 \pm 0,07 metros, $83,4 \pm 11,6 \mathrm{~kg}$ ) alunos do Curso de Monitor de Educação Física 2006 (CMEF) do CBMERJ. Antes da coleta, todos os participantes assinaram o termo de consentimento, de acordo com a resolução $n^{\circ}$. 196/96 do Conselho Nacional de Saúde.

\section{Procedimentos}

Os dados foram coletados em três dias não consecutivos ao longo de uma semana. No primeiro dia, foi realizada uma anamnese geral do avaliado, questionário PAR-Q ${ }^{(9)}$, e questionário para avaliar o ICT ${ }^{(8)}$. Imediatamente após, os indivíduos realizaram as medidas antropométricas. As variáveis antropométricas mensuradas neste estudo foram: massa corporal (MC), em uma balança digital da marca Tanita, modelo TBF-521, com precisão de 100g; estatura, com Antropômetro metálico de Martin, com precisão de $1 \mathrm{~mm}$, tendo sido conferida a sua verticalidade com um fio de prumo. Para estimar a densidade corporal e o percentual de gordura foram utilizadas as equações propostas por e Siri (10) e Jackson e Pollock (11), bem como o índice de massa corporal (IMC) de cada participante.

O segundo dia foi utilizado para a realização dos testes de aptidão musculoesquelética:

Teste de Sentar-e-alcançar (SA) (12): para diminuir a margem de erro durante a coleta, foi colocada uma fita métrica no chão e um pedaço de fita adesiva de 45,7 cm atravessado na marca de $38,1 \mathrm{~cm}$ da fita métrica. $\mathrm{O}$ avaliado sentou-se com a extremidade zero da fita métrica entre as pernas. Os calcanhares deviam quase tocar a fita adesiva na marca dos $38,1 \mathrm{~cm}$ e estarem separados cerca 
Rev. Educ. Fís. 2009 Mar: 144: 36-44. Rio de Janeiro - RJ - Brasil

de $30,5 \mathrm{~cm}$. Com as pernas estendidas, o avaliado inclinou-se para frente lentamente e estendeu as mãos o mais distante possível, permanecendo nesta posição o tempo suficiente para a distância ser marcada. $\mathrm{O}$ avaliador devia segurar os joelhos do avaliado, evitando que estes se flexionem sem os empurrar para baixo. Realizaram-se três tentativas, sendo registrada a melhor das três.

Teste isométrico em barra fixa (BF) (13): para determinar a resistência muscular de membros superiores por meio do teste calistênico isométrico em barra fixa, utilizou-se o seguinte procedimento: a posição da pegada foi pronada e correspondente à distância biacromial (distância entre as mãos devia corresponder à distância entre os ombros). Após assumir essa posição o avaliado deveria elevar o corpo até que o queixo ultrapasse a barra; os braços deviam ser flexionados próximos ao tronco e o peito devia estar o mais próximo possível da barra. $\mathrm{O}$ cronômetro foi acionado no exato momento em que o queixo do avaliado passou acima do nível da barra e foi desativado quando passou abaixo do nível da barra. $\mathrm{O}$ avaliado foi orientado para que realizasse $\mathrm{o}$ máximo de esforço procurando se manter suspenso, com o queixo acima do nível da barra, joelho em extensão, pés fora do solo, o maior tempo possível. Foi registrado o tempo que o avaliado conseguiu manter-se acima do nível da barra.

Teste de flexão dos braços (FB) (14): seguiuse o seguinte protocolo. Os movimentos foram executados com o aluno sobre o solo em decúbito ventral com as pontas dos pés e mãos apoiadas sobre o solo, braços estendidos na linha e largura dos ombros, quadril, joelhos e tronco estendidos. $O$ peito devia tocar o solo a cada movimento de flexão dos braços, e era contada uma repetição após a extensão dos braços e volta à posição inicial. $O$ exercício devia ser feito até a exaustão, contando o número máximo de repetições.

Teste abdominal (TA) (14): teste de flexão do tronco sobre os membros inferiores. Os avaliados foram colocados em decúbito dorsal sobre o solo, com os dedos das mãos tocando a região das têmporas, joelhos flexionados, pés em contato com o solo a aproximadamente $30,5 \mathrm{~cm}$ da região glútea e abertos na largura dos ombros. $\mathrm{O}$ avaliador manteve os pés do avaliado fixos em contato com o solo para não escorregarem; o avaliado, retirando as costas do chão, flexiona o tronco e o quadril até os cotovelos tocarem nos joelhos, voltando à posição inicial com os cotovelos tocando o solo, repetindo o movimento tão depressa e tantas vezes quantas forem possíveis. Marcou-se o número de repetições executadas durante 60 segundos (1 minuto).

Teste de impulsão horizontal (IH) (15): adotou-se o seguinte protocolo: $\mathrm{O}$ avaliado foi colocado com os pés paralelos no ponto de partida, a linha zero da fita métrica fixada ao solo. Ao comando "atenção, já!" O avaliado realizou seu salto no sentido horizontal, com impulsão simultânea das pernas, objetivando atingir o ponto mais distante possível do marco zero. Foi permitida a movimentação livre dos braços. Foram realizadas três tentativas não consecutivas para cada avaliado e registrou-se a marca atingida pela parte anterior do pé mais próxima do ponto de partida.

O terceiro dia de teste foi destinado para o teste de resistência aeróbia (RA): Foi utilizado o Teste de corrida de 2400 metros - 1,5 milha (RA) (18), o protocolo consistiu em caminhar ou correr, continuamente à distância de 1,5 milha no máximo ritmo possível que o aluno testado conseguisse suportar. Ao final do teste, coletou-se o tempo total gasto para completar o percurso.

\section{Tratamento estatístico}

Para análise descritiva, o tratamento estatístico empregado foi de medidas de tendência central e medidas de dispersão, valores mínimos e máximos alcançados. Para verificar o nível de aptidão física foi utilizado o somatório do score-t de todos os testes físicos. Para verificar a correlação entre o somatório do score-t e o ICT foi utilizada a equação de Produto-Momento de Pearson, para tal foi utilizado o programa Estatística versão 7.0. 
RESULTADOS

Os resultados descritivos são apresentados em quatro momentos de acordo com as TABELAS 1, 2, 3 e 4. Na TABELA 1 são apresentados os dados das medidas antropométricas. Na TABELA 2 são apresentados os dados relativos aos testes para as capacidades físicas básicas e o ICT. As TABELAS 1 e 2 descrevem os valores de média, mediana, desvio padrão, mínimo e máximo.

TABELA 1.

DESCRIÇÃO DAS MEDIDAS ANTROPOMÉTRICAS.

\begin{tabular}{cccccc}
\hline & Idade & Estatura & MC & \% G & IMC \\
\hline Média & 33 & 1,79 & 83,4 & 15,1 & 26,1 \\
Mediana & 34 & 1,77 & 80,6 & 15,9 & 25,8 \\
Desv. Pad. & 4 & 0,07 & 11,6 & 2,79 & 2,7 \\
Valor Máximo & 39 & 1,92 & 117,2 & 20,6 & 34,2 \\
Valor Mínimo & 26 & 1,65 & 69,9 & 9,7 & 23,1 \\
\hline
\end{tabular}

Desv. Pad. - desvio padrão; MC - massa corporal; \%G - percentual de gordura; IMC - índice de massa corporal; ICT - índice de capacidade de trabalho.

TABELA 2.

DESCRIÇÃO DOS RESULTADOS DO ICT E DOS TESTES DAS CAPACIDADES FÍSICAS BÁSICAS.

\begin{tabular}{cccccccc}
\hline Indivíduos & ICT & SA & BF & IH & TA & FB & VO $_{2}$ \\
\hline $\mathbf{1}$ & 37 & 37 & 29 & 242 & 38 & 35 & 51,1 \\
$\mathbf{2}$ & 35 & 41 & 75 & 212 & 46 & 45 & 53 \\
$\mathbf{3}$ & 38 & 18,5 & 56 & 258 & 62 & 30 & 46,9 \\
$\mathbf{4}$ & 42 & 17,5 & 17 & 207 & 30 & 10 & 32,7 \\
$\mathbf{5}$ & 32 & 44 & 32 & 241 & 56 & 27 & 51,1 \\
$\mathbf{6}$ & 34 & 26,5 & 47 & 231 & 51 & 35 & 49,3 \\
$\mathbf{7}$ & 39 & 31 & 35 & 217 & 45 & 31 & 52,7 \\
$\mathbf{8}$ & 36 & 25 & 38 & 244 & 42 & 39 & 42,4 \\
$\mathbf{9}$ & 39 & 22,5 & 50 & 258 & 47 & 29 & 40,4 \\
$\mathbf{1 0}$ & 34 & 8 & 22 & 233 & 43 & 39 & 46,8 \\
$\mathbf{1 1}$ & 44 & 45 & 30 & 252 & 49 & 32 & 47,5 \\
$\mathbf{1 2}$ & 41 & 20,5 & 46 & 233 & 53 & 44 & 44,3 \\
$\mathbf{1 3}$ & 33 & 46,2 & 21 & 212 & 42 & 28 & 42,2 \\
$\mathbf{1 4}$ & 31 & 28 & 20 & 234 & 34 & 30 & 44,2 \\
\hline Média & 37 & 29,3 & 37,0 & 233,9 & 45,6 & 32,4 & 46,0 \\
Mediana & 37 & 27,3 & 33,5 & 233,5 & 45,5 & 31,5 & 46,9 \\
Desv.Pad. & 4 & 11,8 & 16,3 & 16,8 & 8,5 & 8,6 & 5,6 \\
Máximo & 44 & 46,2 & 75,0 & 258,0 & 62,0 & 45,0 & 53,0 \\
Mínimo & 31 & 8,0 & 17,0 & 207,0 & 30,0 & 10,0 & 32,7 \\
\hline
\end{tabular}


Na TABELA 3 são apresentados os dados scores-t para cada teste, assim como seu somatório.

Não foram encontradas correlações entre o ICT e nível de aptidão física. O valor encontrado foi de -0,09 para o somatório do score-t de todas as qualidades físicas básicas e o ICT.

$\mathrm{Na}$ TABELA 4 são descritos os percentuais de indivíduos por classificação para cada teste.

\section{DISCUSSÃO}

O propósito do presente estudo foi verificar a correlação entre o ICT e as qualidades físicas básicas, e fornecer dados descritivos sobre o perfil físico de um grupo de integrantes do CBMERJ. Considerando as características dos sujeitos, os resultados do corrente estudo revelaram não haver uma correlação entre o ICT e o nível de aptidão física. Os resultados dos testes de capacidades físicas básicas apresentaram em média bons níveis de aptidão física nos testes de resistência aeróbia, impulsão horizontal, flexão de braços e abdominal, entretanto não ocorreu o mesmo para a flexibilidade e o teste isométrico em barra fixa.

Os correntes resultados apresentaram níveis intermediários para o ICT, 50\% dos bombeiros avaliados apresentaram classificação (moderado), $42 \%$ apresentaram classificação "boa", menos de $10 \%$ apresentaram classificação "excelente" e nenhum indivíduo apresentou baixa capacidade para o trabalho. Por outro lado, Suzuki et al. (16), avaliaram 139 homens metalúrgicos japoneses e encontraram valores superiores aos correntes resultados. Neste estudo, a maior parte dos indivíduos em ambos os grupos apresentaram

TABELA 3.

APRESENTAÇÃO DOS RESULTADOS DOS TESTES DAS CAPACIDADES FÍSICAS BÁSICAS EM SCORE-T.

\begin{tabular}{ccccccccc}
\hline Indivíduos & ICT & SA & BF & IH & TA & FB & VO $_{2}$ & SSTT \\
\hline $\mathbf{1}$ & 50,5 & 56,5 & 45,1 & 54,8 & 41,1 & 53,0 & 59,1 & 309,6 \\
$\mathbf{2}$ & 45,4 & 59,9 & 73,3 & 37,0 & 50,5 & 64,6 & 62,5 & 347,7 \\
$\mathbf{3}$ & 53,1 & 40,8 & 61,6 & 64,3 & 69,3 & 47,2 & 51,5 & 334,8 \\
$\mathbf{4}$ & 63,3 & 40,0 & 37,8 & 34,0 & 31,7 & 24,0 & 26,0 & 193,5 \\
$\mathbf{5}$ & 37,7 & 62,4 & 46,9 & 54,2 & 62,2 & 43,7 & 59,1 & 328,7 \\
$\mathbf{6}$ & 42,9 & 47,6 & 56,1 & 48,3 & 56,4 & 53,0 & 55,9 & 317,2 \\
$\mathbf{7}$ & 55,7 & 51,4 & 48,8 & 40,0 & 49,3 & 48,3 & 62,0 & 299,8 \\
$\mathbf{8}$ & 48 & 46,3 & 50,6 & 56,0 & 45,8 & 57,6 & 43,5 & 299,8 \\
$\mathbf{9}$ & 55,7 & 44,2 & 58,0 & 64,3 & 51,7 & 46,0 & 39,9 & 304,1 \\
$\mathbf{1 0}$ & 42,9 & 31,9 & 40,8 & 49,5 & 47,0 & 57,6 & 51,4 & 278,2 \\
$\mathbf{1 1}$ & 68,5 & 63,3 & 45,7 & 60,8 & 54,0 & 49,5 & 52,6 & 325,9 \\
$\mathbf{1 2}$ & 60,8 & 42,5 & 55,5 & 49,5 & 58,7 & 63,4 & 46,9 & 316,5 \\
$\mathbf{1 3}$ & 40,3 & 64,3 & 40,2 & 37,0 & 45,8 & 44,9 & 43,1 & 275,3 \\
$\mathbf{1 4}$ & 35,2 & 48,9 & 39,6 & 50,1 & 36,4 & 47,2 & 46,7 & 268,8 \\
\hline
\end{tabular}


TABELA 4.

DESCRIÇÃO DA CLASSIFICAÇÃO POR ESCORES DOS INDIVÍDUOS PARA OS TESTES DAS QUALIDADES FÍSICAS BÁSICAS E O ICT.

\begin{tabular}{|c|c|c|c|}
\hline Teste & Classificação & $\begin{array}{c}\text { N}^{\circ} \text { de indivíduos por } \\
\text { classificação }\end{array}$ & $\begin{array}{l}\% \text { de sujeitos por } \\
\text { classificação }\end{array}$ \\
\hline \multirow{4}{*}{ ICT } & Excelente & 1 & 7,1 \\
\hline & Bom & 6 & 42,8 \\
\hline & Moderado & 7 & 50 \\
\hline & Baixo & 0 & 0 \\
\hline \multirow{7}{*}{ SA } & Excelente & 0 & 0 \\
\hline & Bom & 1 & 7,1 \\
\hline & Acima da média & 2 & 14,2 \\
\hline & Média & 1 & 7,1 \\
\hline & Abaixo da média & 1 & 7,1 \\
\hline & Fraco & 4 & 28,5 \\
\hline & Muito fraco & 5 & 35,7 \\
\hline \multirow{5}{*}{ BF } & Excelente & 0 & 0 \\
\hline & Bom & 1 & 7,1 \\
\hline & Média & 0 & 0 \\
\hline & Regular & 4 & 28,57 \\
\hline & Fraco & 9 & 64,29 \\
\hline \multirow{7}{*}{ IH } & Excelente & 10 & 71,4 \\
\hline & Muito bom & 1 & 7,1 \\
\hline & Bom & 3 & 21,4 \\
\hline & Aceitável & 0 & 0 \\
\hline & Regular & 0 & 0 \\
\hline & Fraco & 0 & 0 \\
\hline & Muito fraco & 0 & 0 \\
\hline \multirow{5}{*}{ TA } & Excelente & 11 & 78,5 \\
\hline & Bom & 2 & 14,2 \\
\hline & Média & 1 & 7,1 \\
\hline & Regular & 0 & 0 \\
\hline & Fraco & 0 & 0 \\
\hline \multirow{5}{*}{ FB } & Excelente & 9 & 64,2 \\
\hline & Bom & 4 & 28,5 \\
\hline & Média & 0 & 0 \\
\hline & Regular & 0 & 0 \\
\hline & Fraco & 1 & 7,1 \\
\hline \multirow{6}{*}{$\mathrm{VO}_{2}$} & Superior & 8 & 57,1 \\
\hline & Excelente & 4 & 28,5 \\
\hline & Bom & 1 & 7,1 \\
\hline & Normal & 0 & 0 \\
\hline & Ruim & 1 & 7,1 \\
\hline & Muito ruim & 0 & 0 \\
\hline
\end{tabular}


classificação "boa" e cerca de $20 \%$ classificação "excelente". Zwart, et al., (17) com o objetivo de verificar a reprodutibilidade do ICT proposto por Tuomi ( ${ }^{(8)}$, avaliaram 859 trabalhadores da construção civil alemã. Zwart et al. ${ }^{(17)}$, avaliaram os indivíduos em duas ocasiões, separadas por quatro semanas, e reportaram uma alta reprodutibilidade para o ICT. Novamente foram encontrados valores para ICT superiores aos correntes resultados. Cerca de $80 \%$ dos avaliados obtiveram classificação "excelente" ou "boa" e apenas $14 \%$ dos avaliados apresentaram classificação "moderada". Com base nestes e nos resultados do presente estudo pode-se observar que o ICT variou de acordo com o tipo de trabalho e população. Além disso, o tamanho da amostra pode ter sido um fator determinante sobre os valores de ICT superiores de Suzuki et al. ${ }^{(16)}$ e Zwart et al. ${ }^{(17)}$. Desta forma são necessárias novas pesquisas, com números maiores de participantes, para verificar se o ICT corresponderá adequadamente à aptidão física em bombeiros.

A análise dos resultados das avaliações de aptidão física permite que sejam feitas as seguintes considerações. Segundo a TABELA para Corrida de 1,5 milha $-2,400$ mts ${ }^{(18)}, 78,5 \%$ dos indivíduos avaliados atingiram a escala superior, e 14,2\% atingiram a escala excelente, nenhum avaliado foi classificado como abaixo da normalidade para esse teste. No entanto, é muito difícil comparar nossos resultados com os dos prévios estudos anteriores ${ }^{(3,6,2,7)}$. Apenas Sarah et al., (6) utilizaram o mesmo protocolo do corrente estudo para o teste cardiorespiratório. Neste estudo, através de uma análise retrospectiva, os autores acompanharam o nível das capacidades físicas de estudantes de medicina da universidade de Bethesda, Maryland, sendo todos militares na ativa. Foram feitas duas avaliações anuais, sempre durante o outono e inverno, no período de 2004 a 2006. A média dos tempos encontrados pelos autores foi de 10:54 e 11:31 min:s para o melhor e pior desempenho, respectivamente. O desempenho dos médicos militares foi inferior ao dos bombeiros avaliados no presente estudo, haja vista, que a média do tempo encontrada foi de 10:35 min:s para o mesmo protocolo. Em um estudo similar, Roberts et al., (3) verificaram o volume de oxigênio máximo $\left(\mathrm{VO}_{2 \text { máx }}\right)$, através de um teste submáximo em um cicloergômetro. Para tal, os autores avaliaram recrutas a bombeiros dos Estados Unidos da América (EUA) antes e após 16 semanas do curso de formação. Foram encontrados valores para $\circ \mathrm{VO}_{2 \text { máx }}$ de $35 \pm 7$ e $45 \pm 6(\mathrm{ml} / \mathrm{kg} /$ min-1) antes e após o período de treinamento, respectivamente. Considerando os valores obtidos no corrente estudo $46 \pm 5,6(\mathrm{ml} / \mathrm{kg} / \mathrm{min}-1)$, tais bombeiros aparentemente apresentaram níveis de resistência cardiorrespiratória semelhantes aos bombeiros do EUA, mesmo com protocolos diferentes. Entretanto essa similaridade só ocorreu para os valores encontrados por Roberts et al. (3) pós-treinamento. Outro ponto importante a ser observado é a manutenção da capacidade física pelos bombeiros avaliados no presente estudo, mesmo após o período de formação, os indivíduos apresentam valores similares aos bombeiros recém formados.

Para verificar a resistência muscular foram utilizados dois testes, FB e TA. Analisando os escores obtidos pelos militares deste estudo no teste $\mathrm{FB}$, nota-se que a maioria dos indivíduos encontrase entre a classificação "boa" $(28,5 \%)$ e "excelente" (64,2\%), de acordo com a TABELA proposta por Pollock e Wilmore ${ }^{(14)}$. Os valores encontrados no presente estudo foram superiores aos encontrados por Pereira e Teixeira ${ }^{(2)}$, que avaliaram 1014 indivíduos, sendo 985 homens, todos militares da Aeronáutica de uma Unidade Militar do sul do Brasil. Os autores encontraram uma média de $22,03 \pm 7,4$ para os militares do sexo masculino, valores inferiores ao observado no corrente estudo $32 \pm 8,6$ FB. O mesmo não ocorreu para o TA, os valores encontrados por Pereira e Teixeira (2) foram similares ao do presente estudo. Os militares da aeronáutica obtiveram uma média de 40,7 \pm 10,3 
e os bombeiros $46 \pm 8,5$ no TA, a diferença entre ambos os grupos foi menor que o desvio padrão de cada grupo. Outra qualidade física verificada foi a potência de membros inferiores. Para verificá-la foi adotado o teste de $\mathrm{IH}$, e novamente a maior parte dos avaliados obtiveram altos escores e foram classificados como "excelente", através da TABELA descrita por Fernandes ${ }^{(19)}$. Diante de tais achados, parece existir uma convergência do perfil físico do bombeiro para uma boa aptidão musculoesquelética. Em todos os testes propostos para medir essa característica, a amostra em média apresentou valores superiores às TABELAS de normalidade.

Através do teste SA foi verificada uma deficiência pelos bombeiros avaliados no corrente estudo para flexibilidade, segundo a classificação de Morrow et al., (20), mais da metade dos avaliados possuíam níveis de flexibilidade "fraca" $(28,5 \%)$ e "muito fraca" $(35,7 \%)$. Todavia, esse tipo de profissional parece não necessitar de altos níveis de flexibilidade durante o cotidiano de seu trabalho. Outra capacidade física revelada deficiente nos bombeiros do CBMERJ foi a força isométrica avaliada através do teste de BF. Cerca de $90 \%$ dos avaliados se encontraram entre as classificações regular e fraco, segundo a TABELA disponibilizada por Marins (21). Analisando os escores obtidos pelos indivíduos avaliados no corrente estudo, verificamos um menor rendimento para as qualidades físicas mensuradas de forma estática. Os dois únicos testes que possuem essa característica SAe BF apresentaram valores abaixo dos escores das TABELAS de normalidade ${ }^{(20,21)}$. Contraditoriamente, os indivíduos apresentaram boas classificações para as qualidades físicas avaliadas de forma dinâmica IH, FB, FA e RA. Baseado nos correntes resultados pode-se observar que o bombeiro necessita em sua atividade profissional de capacidades físicas que estejam relacionadas a trabalhos dinâmicos.

Uma limitação do presente estudo que pode ser destacada se refere ao tamanho da amostra utilizada. O " $n$ " amostral não é suficiente para extrapolarmos tais resultados para a população em questão (servidores do CBMERJ). Portanto, novas pesquisas são necessárias, a fim de verificar a relação entre as capacidades físicas básicas e o ICT em um maior número de bombeiros.

\section{CONCLUSÃO}

O ICT não apresentou uma forte correlação com as qualidades físicas básicas em bombeiros do CBMERJ. Os avaliados apresentaram bons níveis de resistência, potência muscular e resistência cardiorrespiratória, embora também tenha apresentado baixos níveis de flexibilidade e força isométrica, o que resultou na classificação moderada no ICT para a maioria dos participantes. O ICT não mostrou ser um método adequado para quantificar a capacidade de trabalho nos profissionais que dependem do desempenho físico para executar suas atividades diárias, tendo em vista que tais profissionais apresentaram altos níveis de condicionamento físico e apenas classificações intermediárias para o ICT.

\section{AGRADECIMENTOS}

O Prof. Dr. Roberto Simão agradece ao CNPQ - Bolsa Produtividade e a FAPERJ - Auxílio Instalação.

\section{REFERÊNCIAS BIBLIOGRÁFICAS}

1. Freitas RG, Filho JF. Perfis dermatoglíficos, somatotípico, das qualidades físicas da força e velocidade de reação, VO2máx e da coordenação motora, características de pilotos de helicópteros da Força Aérea Brasileira (FAB). Fitness \& Performance Journal 2003;3:115-20.

2. Pereira EF, Teixeira CS. Proposta de valores normativos para avaliação da aptidão física em militares daAeronáutica. Revista Brasileira Educação Física Esporte 2006;20:249-56.

3. Roberts MA, O'dea J, Boyce A, Mannix ET. Fitness levels of firefighter recruits before and after a supervised exercise training program. Journal Strength Conditioning Research 2002;16:271-7.

4. Sampaio AO, Dantas PMS, Dias AC, Fernandes FJ. Perfis somatotípicos, de qualidades físicas básicas e 
dermatoglífico dos pilotos de caça da FAB, com patentes de tenente e capitão. Fitness \& Performance Journal 2003;2:122-8.

5. Santos MR, Filho JF. Perfis dermatoglíficos, somatotípicos e das qualidades físicas básicas dos pára-quedistas de Exército Brasileiro do ano de 2003. Fitness \& Performance Journal 2004;3:88-99.

6. Sarah D, Mitchell BA, Richard Eide BA, Cara H, Olsen DRPH, Mark B, Stephens, MD. Body Composition and Physical Fitness in a Cohort of US Military Medical Students. Journal of American Board of Family Medicine 2008;21:165-7.

7. Boldori R. Aptidão física e sua relação com a capacidade de trabalho dos bombeiros militares do estado de Santa Catarina. Dissertação apresentada ao Programa de PósGraduação em Engenharia de Produção da Universidade Federal de Santa Catarina; Florianópolis: UNISUL, 1999.

8. Tuomi K, Idlmarinen J, Jahkola A, Katajarinne L, Tulkki A. Work ability index. Occupational Health Care 19. (Finnish Institute of Occupational Health, Helsinki), 1997.

9. Shephard RJ. PAR-Q, Canadian home fitness test and exercise screening alternatives. Sports Medicine 1988;5:185-95.

10. Siri WE. Body composition from fluid space and density. In: Brozek J, Henschel A, editors. Techniques for measuring body composition. Washington DC: National Academy of Science 1961;223-4.

11. Jackson AS, Pollock ML. Generalized equations for predicting body density of men. Br J Nutr 1978;40:497504.

12. Johnson, BL, Nelson JK. Practical Measurements for Evaluation in Physical Education. Minnesota: Burgess Publishing Company, 1979.

13. Heyward VH, Stolarczyk LM. Avaliação da composição corporal aplicada. São Paulo: Manole, 2000.

14. Pollock ML, Wilmore JJ, Fox SM. Exercícios na saúde e na doença. Rio de Janeiro: Medsi, 1993.

15. Soares J, Sessa M. Medidas da força muscular. In: Matsudo VKR, editor. Testes em Ciências do Esporte. 4th ed. São Caetano do Sul: CELAFISCS, 1987: 57-68.

16. Suzuki H, Kumashiro M, Kusano K, Shazuki S, Fuji A, Erro R. Comparison of work ability índex and congnitive function tests. Journal of Occupational Health 2004;
46:71-7.

17. Zwart B, Frings-Dresen W, Duivenbooden J. Testrestest reability of the work ability index questionnaire. Occupational and Environmental Medicine 2002;4:17781.

18. Cooper Institute for Aerobic Research. Fitnessgram. Manual de aplicação de testes. Lisboa: Faculdade de Motricidade Humana. 2002.

19. Fernandes FJ. Impressões dermatoglíficas: marcas genéticas na seleção dos tipos de esporte e lutas (o exemplo do desportista do Brasil). 1997. Tese doutorado, Moscou, URSS.

20. Morrow JRJ, Allen WJ, James GD, Dale PM. Measurement and evaluation in human performance. Champaign: Human Kinetics,1995.

21. Marins JCBE, Giannichi RS. Avaliação e Prescrição de Atividade Física - Guia Prático, $2^{\text {nd }}$ ed. Rio de Janeiro: Shape, 1998.

\section{Endereço:}

Cristiano Marcelino

Escola de Educação Física e Desportes (LABOFISE)

Universidade Federal do Rio de Janeiro

Av Carlos Chagas Filho. Cidade Universitária - Ilha do Fundão.

Rio de Janeiro, CEP - 21941-590. Brasil.

cmarcelino@ufrj.br 